\title{
VERIFICATION OF ANALYTICAL MODELS OF THE S-N CURVE WITHIN LIMITED FATIGUE LIFE
}

\author{
Przemyseaw Strzelecki, Janusz Sempruch \\ University of Science and Technology, Faculty of Mechanical Engineering, Bydgoszcz, Poland \\ e-mail:p.strzelecki@utp.edu.pl; semjan@utp.edu.pl
}

\begin{abstract}
This paper presents analytical methods for determination of the curve for a high-cycle fatigue. It has been found based on qualitative and quantitative verification that the error introduced by these methods can be as large as 3-fold length of the experimental life. In addition, the wrong result can lay on either the safe or the unsafe side with equal probability. Therefore, an analytical-and-experimental (hybrid) method has been proposed. Verification of computed characteristics vs. experimental data demonstrated that the "proprietary" proposal reduced the error. In addition, an approximate error depending on the number of experiments has been determined based on the computations.
\end{abstract}

Keywords: fatigue design, S-N curve, high-cycle fatigue, accelerated method

\section{Introduction}

While designing a new component of a machine, the designer has to give it correct dimensions. Typically, the part is exposed to loads variable in time, which can produce fatigue failure. This is why the fatigue life of the component is determined by means of computations. Before the computations can be made, fatigue characteristics of the material or component has to be available, such as that provided by Skibicki et al. (2012). Because this information is typically unavailable at the preliminary computation phase, analytical methods are used to determine these characteristics based on static properties of the material or based on characteristics available for a test specimen of different geometry, an example of which can be found in Tomaszewski et al. (2014). The references describe many such methods but their authors fail to describe the possible error inherent to the method. Note further that the resulting fatigue characteristics predict the point of destruction of the component with a $50 \%$ probability whereas, for design purposes, engineers use plots featuring a $95 \%$ or higher probability factor. An appropriate coefficient is used to bring the fatigue characteristics to the required level of reliability. The following points describe a method for determining this coefficient.

The approach described in the FITNET procedure, documented in the report by Kocak et al. (2006) is one of the latest analytical methods used to determine fatigue characteristics. The main assumption underlying the algorithm is the determination of the fatigue limit for a material consisting of multiplication of the material tensile strength by an appropriate coefficient. The basic number of cycles adopted for the method is $10^{6}$ cycles (same as in the remaining methods described in the literature). However, for the limited life, the method uses slope coefficients $m=5$ for normal stress and $m=8$ for shear stress. See a diagram of the algorithm in Fig. 1a.

Another of the discussed methods is the one proposed by Lee et al. (2005). This approach assumes that 2 points on the plot are required for the determination of fatigue characteristics: the fatigue limit for the basic $10^{6}$ cycles for steel and the fatigue for $10^{3}$ cycles. See Fig. 1b for the illustration of the method. 
(a)

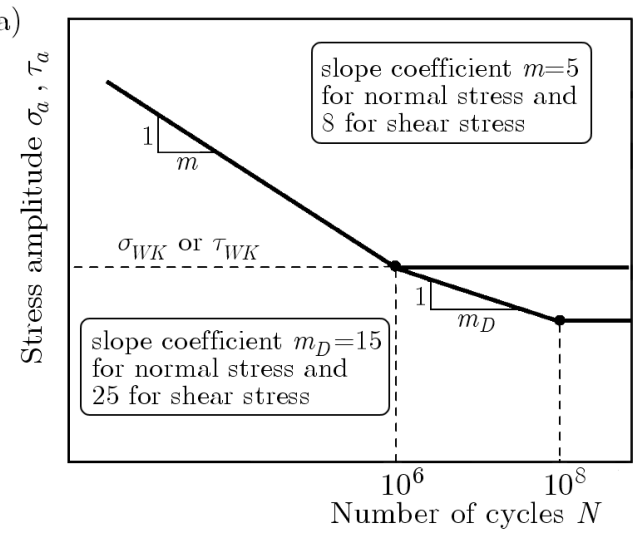

(c)

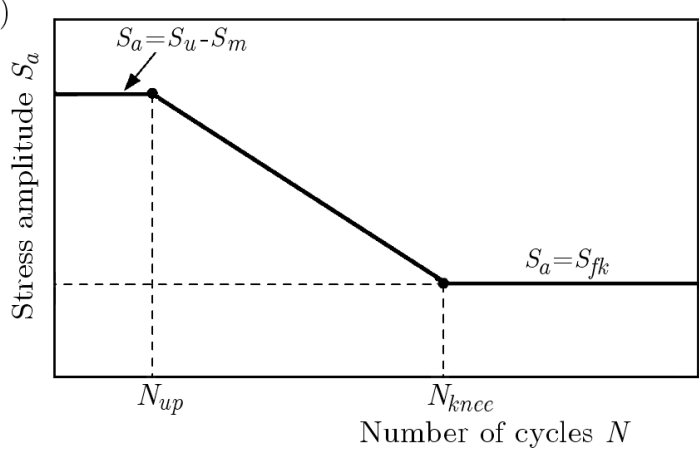

(b)

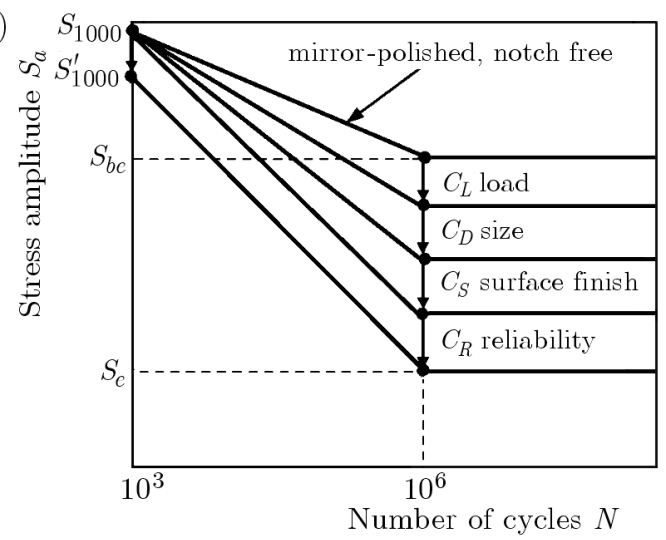

(d)

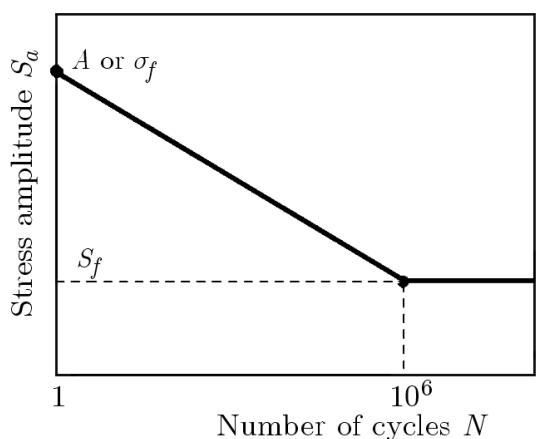

Fig. 1. S-N characteristics according to: (a) FITNET method, (b) Lee and Taylor's method, (c) Schijve's method and (d) method by Stephens et al. (2001)

Another approach described in the literature is the one proposed by Schijve (2009). As in the previous method, again, 2 points are required for the determination of fatigue characteristics: the fatigue limit $S_{f k}$ for the basic $10^{6}$ cycles $\left(N_{\text {knee }}\right)$ for steel and the fatigue life for $10^{2}$ cycles $\left(N_{u p}\right)$. The author of the method claimed that the value adopted as the material tensile strength $S_{u}$ less than the mean stress $S_{m}$ was a good approximation. See Fig. 1c for a schematic description of the procedure.

One more approach discussed in this paper is the method proposed by Stephens et al. (2001). It is based on setting 2 points: the fatigue life for an unlimited life $S_{f}$ and the strength for one loading cycle $A$. The value of $A$ can be determined experimentally using Basquin's equation or be adopted as the value of the actual tensile strength $\sigma_{f}$ (breaking force on elongation divided by the minimum cross sectional area on rupture). If the foregoing information is not available, $A$ can be equal to the material tensile strength (see Fig. 1d) for a schematic procedure for the estimation of the fatigue characteristics.

The last of the presented methods is the "proprietary" one. In this approach, the determination of the fatigue limit is based on the method described in the FITNET procedures while the value of the straight line slope coefficient within the limited life range is computed as follows

$$
m_{e \sigma}=\frac{\log \frac{10^{6}}{N_{R e}}}{\log \frac{0.9 R_{e}}{\sigma_{W K}}} \quad N_{R e}=400\left(\frac{R_{e}}{R_{m}}\right)^{-10}
$$

where $R_{m}$ is tensile strength, $R_{e}$ - yield point.

The proprietary method of analytical determination of the Wöhler diagram is shown in Fig. 2. See the paper by Strzelecki and Sempruch (2012) for a more detailed description.

The solid line represents the fatigue plot for the $50 \%$ probability and the dashed line defines the characteristics for the target probability. A plot featuring the probability different than $50 \%$ is obtained by multiplying the fatigue limit $\left(\sigma_{W K}, \tau_{W K}\right)$ by the coefficient $C_{R}$ (Table 1 ). 


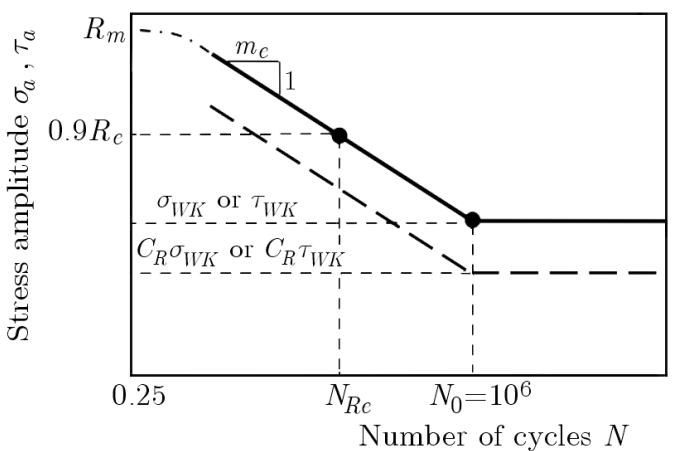

Fig. 2. S-N characteristics according to the proprietary method

Table 1. Value of the coefficient of reliability for different levels of reliability

\begin{tabular}{|c|c|}
\hline Reliability & $C_{R}$ \\
\hline \hline 0.9 & 0.897 \\
\hline 0.95 & 0.868 \\
\hline 0.98 & 0.836 \\
\hline 0.99 & 0.814 \\
\hline 0.999 & 0.753 \\
\hline 0.9999 & 0.702 \\
\hline
\end{tabular}

The method for obtaining these values is described in the paper by Strzelecki and Sempruch (2013). Note that the values of $C_{R}$ obtained by the authors are based on the normal distribution of the fatigue limit and the coefficient of variation is equal to 0.08 (value proposed in the literature).

\section{Analytical verification of methods for approximate determination of the characteristics}

Because the literature is silent on the error made while using the analytical methods described in the foregoing Section, the authors decided to verify these algorithms. The qualitative verification was based on the determination whether the estimated characteristics fits within the safe zone or not. See Fig. 3 for a sample diagram used for the evaluation of these methods.

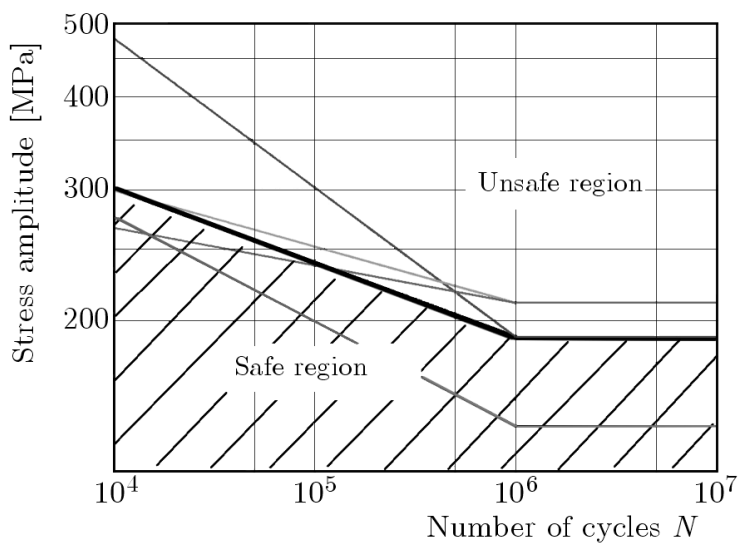

Fig. 3. Diagram for steel S235JR (Robak et al., 2012) (black line) vs. characteristics obtained by analytical methods: sample diagram illustrating the qualitative evaluation of the characteristics

Where the estimated characteristic was positioned within the safe zone (the dashed part of the diagram), the estimated life of the material was identified with the "+" sign. Otherwise, the 
sign was negative ("-"). However, if the characteristics laid on the safe side but shifted to the unsafe side, it was marked with the "+/-" signs. If vice versa, the signs was reversed to "-/+". For the "+/-" case, the fatigue limit was found to lay on the safe side but the slope coefficient of the analytical characteristics was smaller than the value determined based on the experimental data. But when the case was flagged with the " $-/+$ " signs, the method overestimated the fatigue limit, but the resulting slope coefficient was larger than the experimental value. See Table 2 for the results of verification for smooth samples made of 71 grades of steel.

Table 2. Results of qualitative verification of analytical methods

\begin{tabular}{|c|c|c|c|c|c|}
\hline \multirow{2}{*}{$\begin{array}{c}\text { Evaluation } \\
\text { sign }\end{array}$} & \multicolumn{5}{|c|}{ Method } \\
\cline { 2 - 6 } & FITNET & Lee \& Taylor & Schijve & Stephens et al. & Proprietary \\
\hline \hline+ & $6 / 8 \%$ & $21 / 30 \%$ & $17 / 24 \%$ & $28 / 39 \%$ & $32 / 45 \%$ \\
\hline$+/-$ & $33 / 46 \%$ & $20 / 28 \%$ & $3 / 4 \%$ & $0 / 0 \%$ & $9 / 13 \%$ \\
\hline$-/+$ & $0 / 0 \%$ & $6 / 8 \%$ & $8 / 11 \%$ & $26 / 37 \%$ & $6 / 8 \%$ \\
\hline- & $32 / 45 \%$ & $24 / 34 \%$ & $43 / 61 \%$ & $17 / 24 \%$ & $24 / 34 \%$ \\
\hline
\end{tabular}

In order to verify the procedural algorithm in quantitative terms, the authors determined the strength of the specified material for the life of $10^{5}$ cycles based on the fatigue characteristics taken from the literature and, then, determined the material life for the known stress based on the characteristics determined using the specified method. See Fig. 4 for an illustration of the procedure.

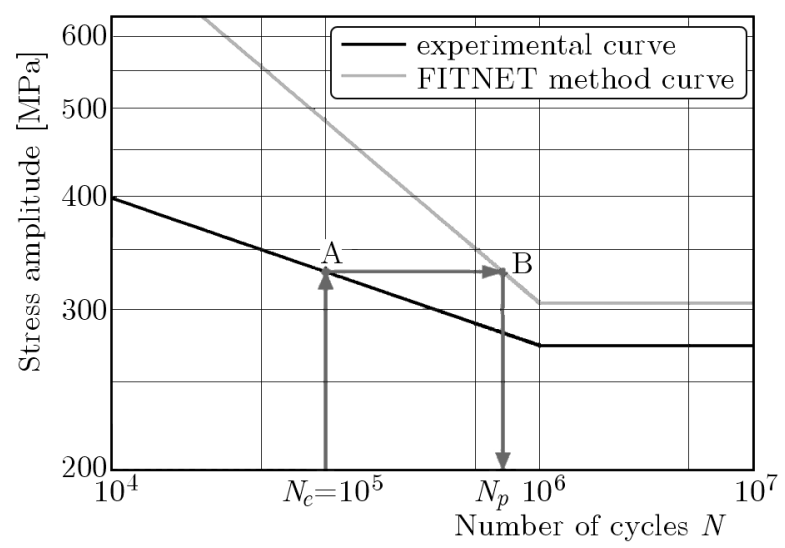

Fig. 4. Fatigue plot for steel S355J0 (Ligaj and Szala, 2010) and fatigue plot account to FITNET: presentation of the method of computation of the estimation error of the analytical method

The error was computed using the difference between the logarithmized life obtained for the experimental characteristics $\left(N_{e}-\right.$ value for $10^{5}$ cycles) and the logarithmized life determined using the analytical method $N_{p}$. The determination of this value was written with

$$
B_{l}=\log N_{e}-\log N_{p} \quad B_{l}=\log \frac{N_{e}}{N_{p}}
$$

Note that there were cases when the value of the fatigue limit determined by the analytical method was larger than the material strength for $10^{5}$-cycle life based on the experimental characteristics. In this case, the straight line from the limited life range was extended until it provided the target stress value. In such a case, the life $N_{p}$ was longer than $10^{6}$ cycles.

Based on the value of the error computed using equation $(2.1)_{2}$, the authors determined the normal distribution (i.e., mean and standard deviation) for this error for each of the methods listed in Table 3. In addition, the table presents the results of the Shapiro-Wilk test for the normality of distribution performed using application $R$, v. 2.15.3, 64-bit. 
Table 3. Quantitative verification results

\begin{tabular}{|c|c|c|c|c|}
\hline \multirow{2}{*}{ Method } & \multicolumn{2}{|c|}{$\begin{array}{c}\text { Normal distribution } \\
\text { values }\end{array}$} & \multicolumn{2}{c|}{$\begin{array}{c}\text { alues obtained by the } \\
\text { Shapiro-Wilk test }\end{array}$} \\
\cline { 2 - 5 } & $\mu$-mean & $\sigma$-SD & & $\mu$-mean \\
\hline \hline FITNET & -0.4874 & 0.3920 & 0.9838 & 0.4744 \\
\hline Lee \& Taylor & -0.1603 & 0.8597 & 0.9725 & 0.1208 \\
\hline Schijve & -0.5201 & 1.0915 & 0.9817 & 0.3888 \\
\hline Stephens et al. & 0.0767 & 1.5589 & 0.9854 & 0.5821 \\
\hline Proprietary & 0.1676 & 0.8574 & 0.9814 & 0.3760 \\
\hline
\end{tabular}

To illustrate the distribution of the error generated by each method, the distributions were overlaid on the plot of the density of probability (Fig. 5).

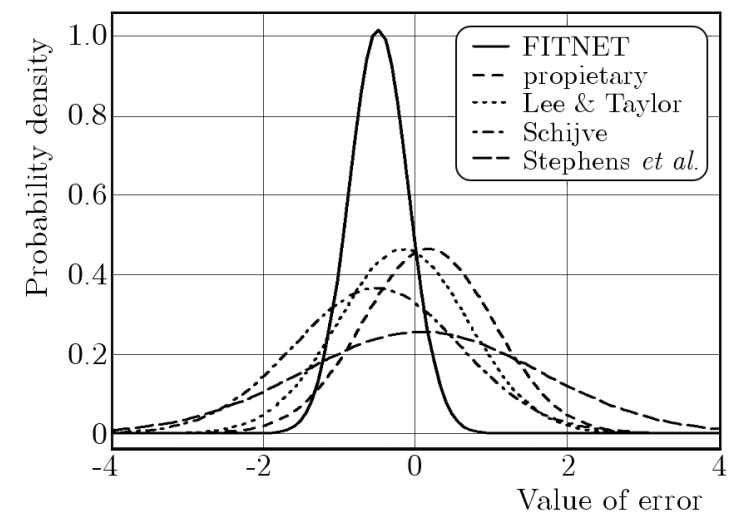

Fig. 5. Plot of the function of density of errors for the individual methods

\section{Analytical and experimentala metod}

Considering that the qualitative and quantitative verification presented in Section 2 showed that the analytical methods can generate significant errors, the authors decided to propose a hybrid solution. It consists of setting the characteristics using an analytical method and, then, correcting the accuracy of life determination by carrying out a "simplified" experiment. This simplified experiment consists of determining an experimental point within the limited life range for the strength corresponding to $10^{5}$ cycles based on the analytical characteristics. The schematic procedure is illustrated in Fig. 6. Note that 3 fatigue tests were carried out and arithmetic mean was computed for the tests to determine the point in support of the analytical method.

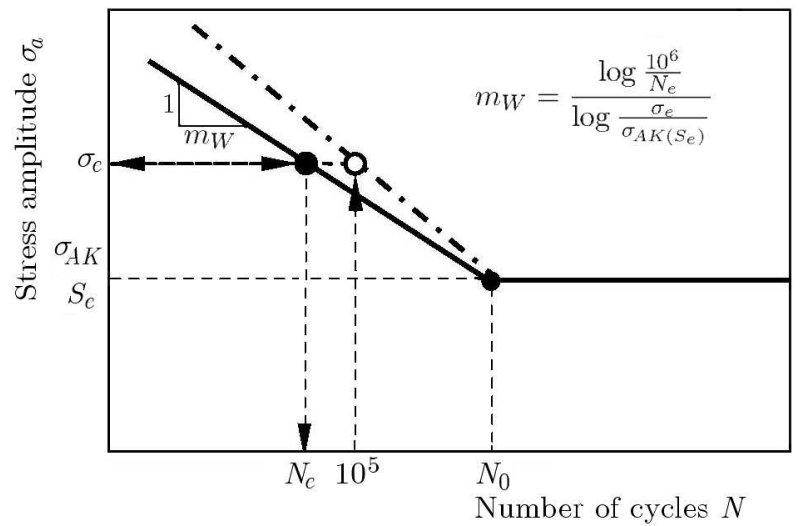

Fig. 6. Schematic presentation of the experimental support for the analytical method 


\section{Experimental verification of the proposals}

To verify the proposals presented in the foregoing sections, the authors carried out an experiment consisting of plotting the fatigue of materials $\mathrm{C} 45+\mathrm{C}$ (as delivered) and $42 \mathrm{CrMo} 4$ (toughened). The static properties of the materials are presented in Table 4. The fatigue properties under high-cycle loading were determined using a device for rotating bending based on a proprietary design presented and verified in the authors' paper (2012).

Table 4. Static properties of the materials tested

\begin{tabular}{|c|c|c|}
\hline \multirow{2}{*}{ Property } & \multicolumn{2}{|c|}{ Material } \\
\cline { 2 - 3 } & C45+C & 42 CrMo4 \\
\hline \hline$R_{m}[\mathrm{MPa}]$ & 826 & 1172 \\
\hline$R_{e}[\mathrm{MPa}]$ & 647 & 1095 \\
\hline $\mathrm{HRC}$ & 21.1 & 32.7 \\
\hline
\end{tabular}

The tests were conducted on smooth and circumferentially notched samples. The drawings of the samples are shown in Fig. 7 and the diagrams based on the data obtained from the experiments are presented in Fig. 8.

(a)

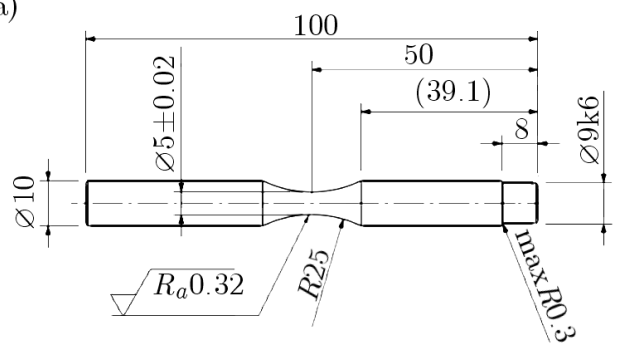

(b)

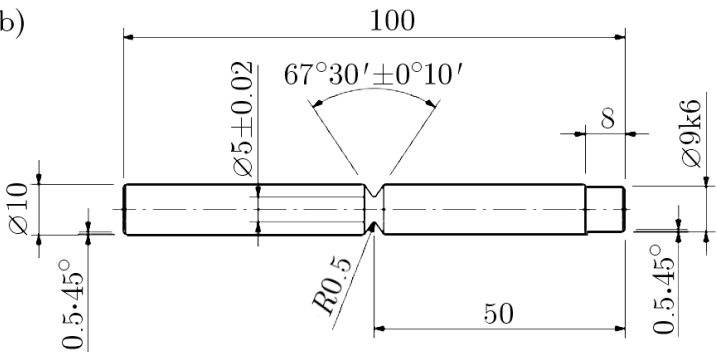

Fig. 7. Test samples: (a) smooth, (b) notched

\section{Verification results}

To verify the accuracy of application of the reliability factor described in Section 1 used to generate the fatigue plot by the analytical method for the required level of probability, Fig. 9 presents plots obtained for the experimental data and characteristics obtained by the analytical method for the $50 \%$ and $95 \%$ probabilities of survival.

The analytical and hybrid methods were verified in accordance with the methodology described by Park and Song (1995). The following equations (Park and Song, 1995) are used to estimated the quantitative conformity of the analytical characteristics to the experimental plot

$$
\begin{aligned}
& E_{f}(s)=\frac{\frac{1}{s} \leqslant \frac{N_{p}}{N_{f}} \leqslant s}{n} \\
& E_{a}=\frac{(1-|\alpha|)+(1-|1-\beta|)+(1-|1-\alpha-\beta|)+(1-|1-r|)}{4}
\end{aligned}
$$

where: $N_{p}$ - life obtained by the application of the verified characteristics, $n$ - number of experimental points, $\alpha$ - free term in the equation for simple regression for the verified method, $\beta$-slope coefficient for the regression line for the verified method, $r$ - correlation coefficient for the regression line for the verified method. 
(a)

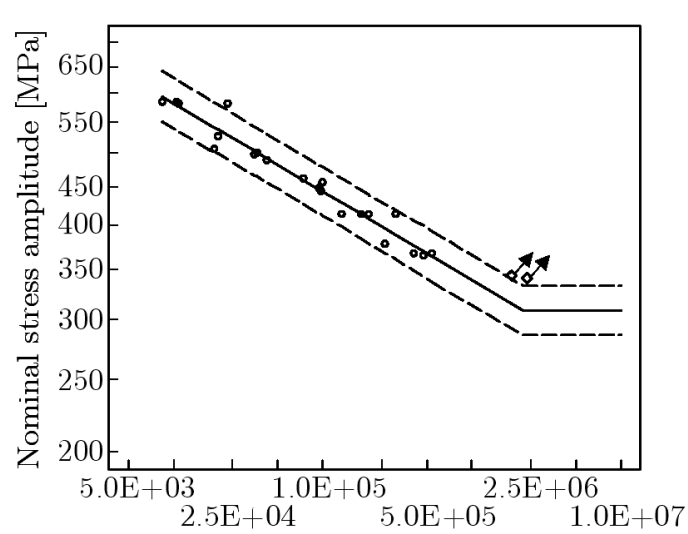

(c)

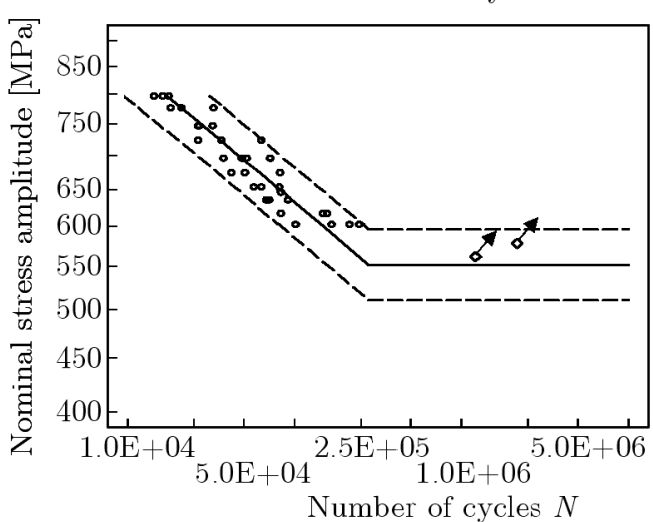

(b)

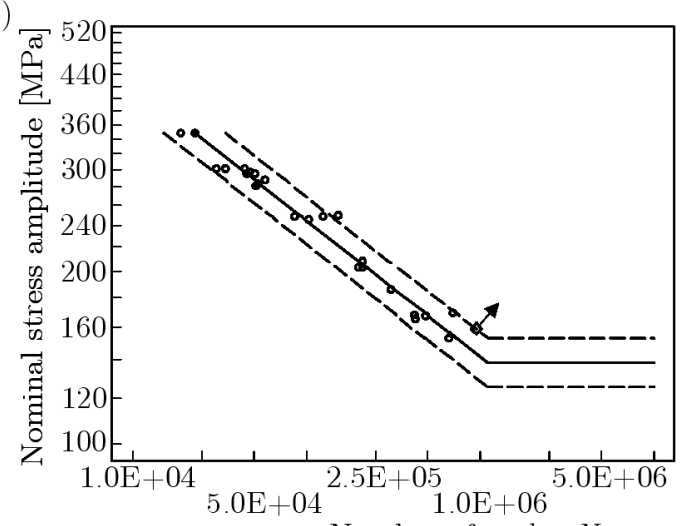

(d)

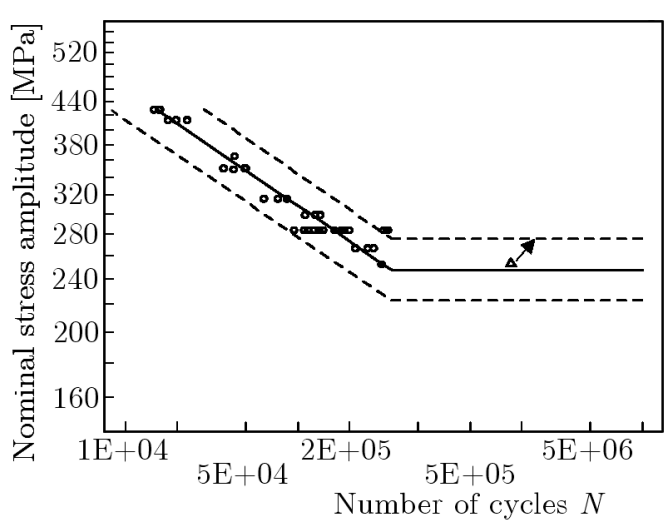

Fig. 8. The resulting fatigue characteristics for: (a) material C45+C, smooth sample,

(b) material $\mathrm{C} 45+\mathrm{C}$, notched sample, (c) material 42CrMo4, smooth sample, (d) material 42CrMo4, notched sample

The value of $E_{f}(s)$ ranges from 0 to 1 and stands for the number of points determined by the analytical method, located within the specified scatter band. On the other hand, the maximum value of $E_{a}$ is 1 but the quantity has no lower limit. The last relation defines the matching of the straight line estimated from the points obtained by the analytical method to the ideal straight line assuming that the experimental life is equal to the estimated life. Table 5 shows the results of computations for the foregoing analytical methods.

For instance, Fig. 10 shows the subsequent plots based on the FITNET method and on the experimental characteristics.

The following equation (Jakubiec and Malinowski, 1996) was used to perform statistical analysis aiming at the determination of the error made using the analytical method and the analytical-experimental (hybrid) method based on experimental values, i.e., the measurement of hardness, tensile strength $R_{m}$, yield point $R_{e}$, life determined within the limited strength range (mean from 3 measurements) and fatigue limit determined with the LOCATI method

$$
B_{p}=\sqrt{\left(\frac{\partial f}{\partial x_{1}}\right)^{2} \Delta x_{1}^{2}+\left(\frac{\partial f}{\partial x_{2}}\right)^{2} \Delta x_{2}^{2}+\cdots+\left(\frac{\partial f}{\partial x_{n}}\right)^{2} \Delta x_{n}^{2}}
$$

where: $f$ - equation of the function defining the value of the quantity being determined, $\Delta x_{n}-$ standard deviation of the $n$-th measured value, $x_{n}-n$-th quantity measured in an intermediate measurement. 
(a)

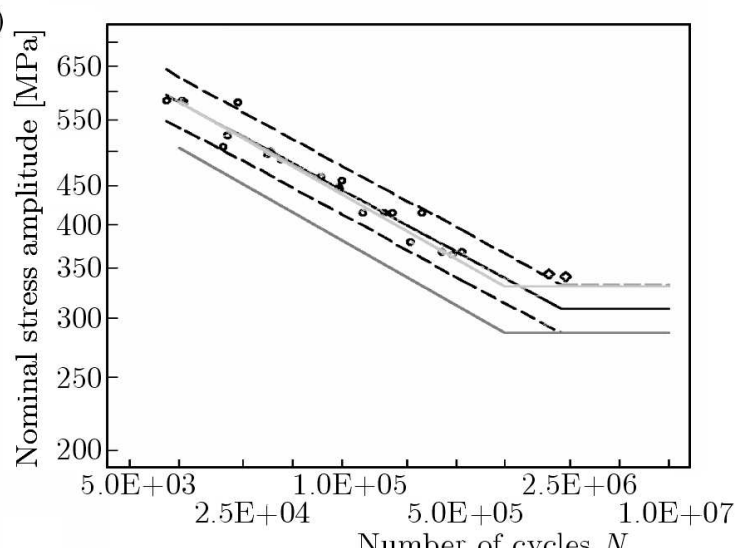

(c)

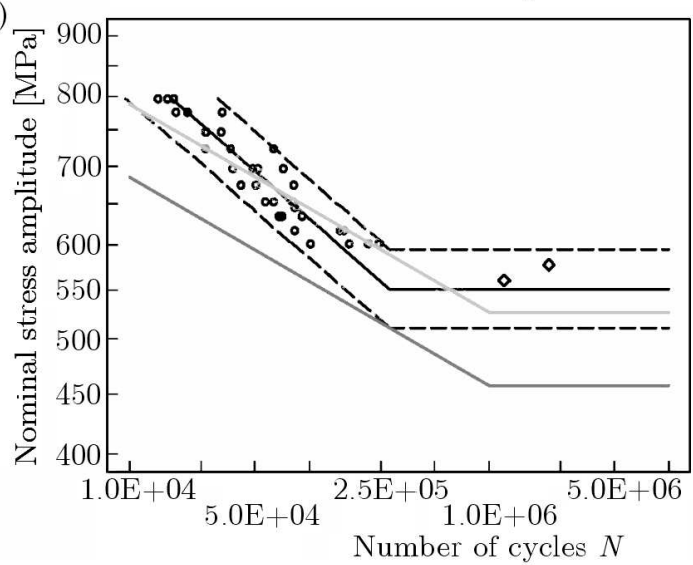

(b)

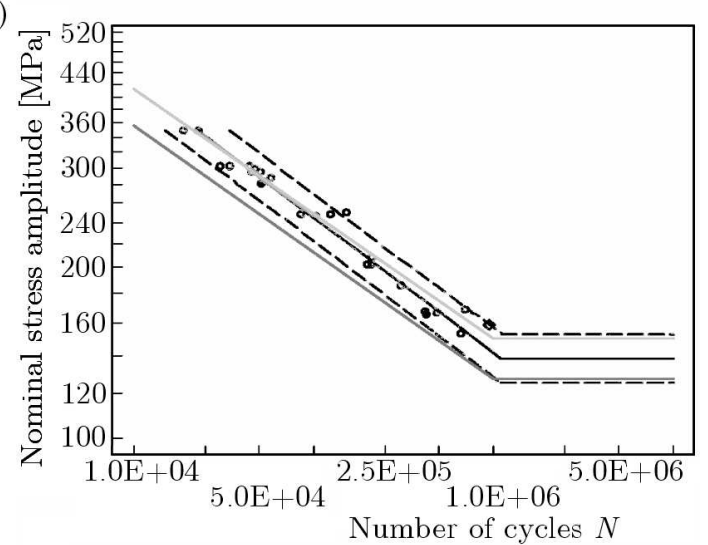

(d)

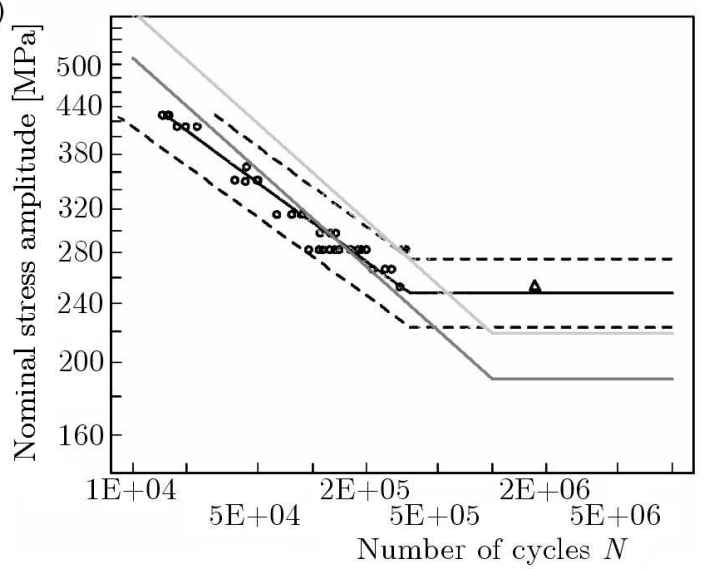

Fig. 9. Fatigue plots: experimental (black line), analytical (grey line) and set off to the $95 \%$ reliability level for the analytical method (dark grey line) for: (a) material $\mathrm{C} 45+\mathrm{C}$, smooth sample,

(b) material $\mathrm{C} 45+\mathrm{C}$, notched sample, (c) material 42CrMo4, smooth sample, (d) material 42CrMo4, notched sample

Table 5. Values of coefficients $E_{f}$ and $E_{a}$ for the analytical and analytical-experimental methods for the tested materials

\begin{tabular}{|c|c|c|c|c|c|c|c|c|c|}
\hline \multirow[b]{2}{*}{$\begin{array}{c}\text { Material } \\
\text { type }\end{array}$} & \multicolumn{9}{|c|}{ Method } \\
\hline & 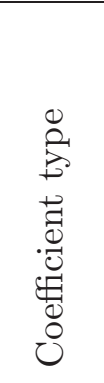 & 点 & 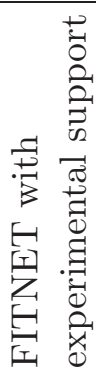 & 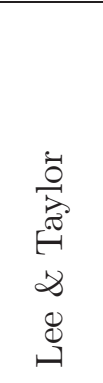 & 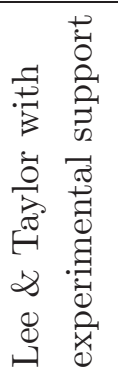 & 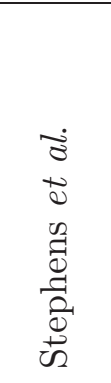 & 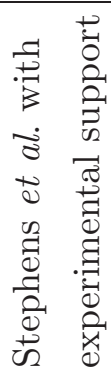 & $\sum_{\substack{: \\
\hdashline}}^{0}$ & 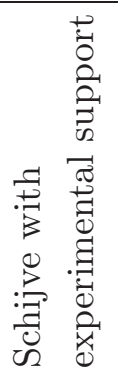 \\
\hline \multirow[t]{2}{*}{$\mathrm{C} 45+\mathrm{C}$} & $E_{f}(3)$ & 0.64 & 1.0 & 0.27 & 0.46 & 0.95 & 0.95 & 0.41 & 0.46 \\
\hline & $\overline{E_{a}}$ & -0.34 & 0.75 & 0.09 & -0.83 & 0.46 & 0.73 & -0.37 & -0.83 \\
\hline \multirow[t]{2}{*}{$42 \mathrm{CrMo} 4$} & $E_{f}(3)$ & 0.09 & 1.0 & 0.06 & 0.36 & 0.82 & 1.0 & 0.58 & 0.58 \\
\hline & $E_{a}$ & -0.91 & 0.7 & -0.71 & -2.38 & 0.41 & 0.79 & -0.38 & -0.37 \\
\hline \multirow{2}{*}{$\begin{array}{c}\mathrm{C} 45+\mathrm{C} \\
\text { notched }\end{array}$} & $E_{f}(3)$ & 0.0 & 0.39 & 0.44 & 0.56 & 0.5 & 0.56 & 0.5 & 0.56 \\
\hline & $E_{a}$ & 0.22 & -2.2 & -0.57 & -2.1 & -1.33 & -2.46 & -0.81 & -2.10 \\
\hline \multirow{2}{*}{$\begin{array}{c}42 \mathrm{CrMo} 4 \\
\text { notched }\end{array}$} & $E_{f}(3)$ & 0.0 & 0.0 & 0.0 & 0.2 & 0.23 & 0.34 & 0.09 & 0.4 \\
\hline & $E_{a}$ & -0.95 & -1.6 & 0.05 & -1.46 & -0.05 & -0.57 & 0.26 & -0.35 \\
\hline
\end{tabular}


(a)

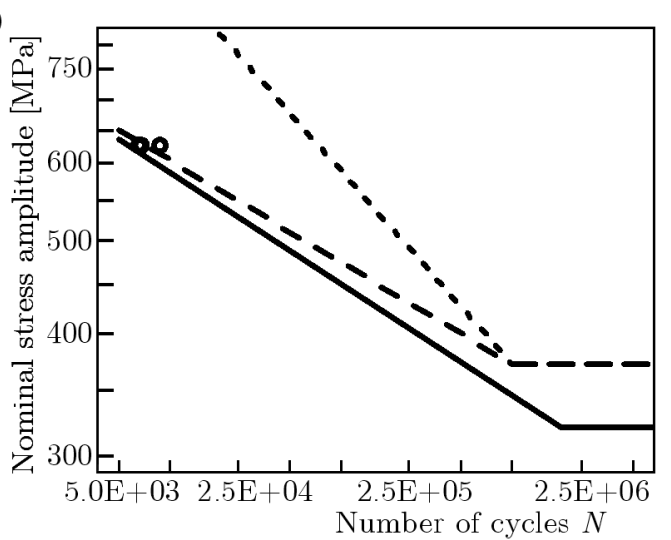

(c)

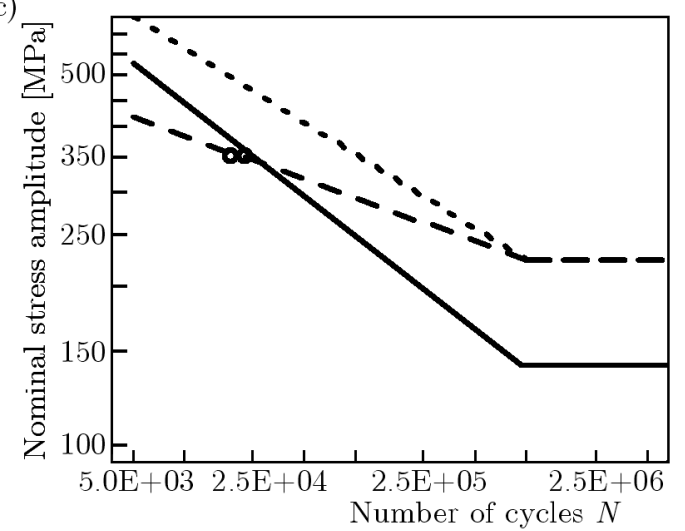

(b)

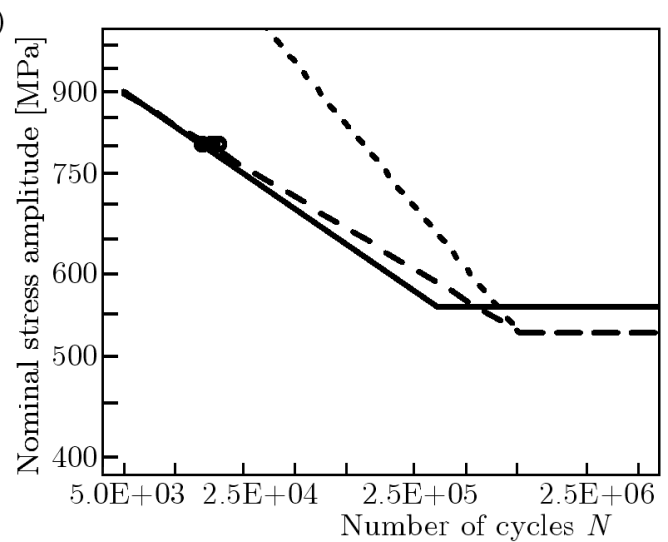

(d)

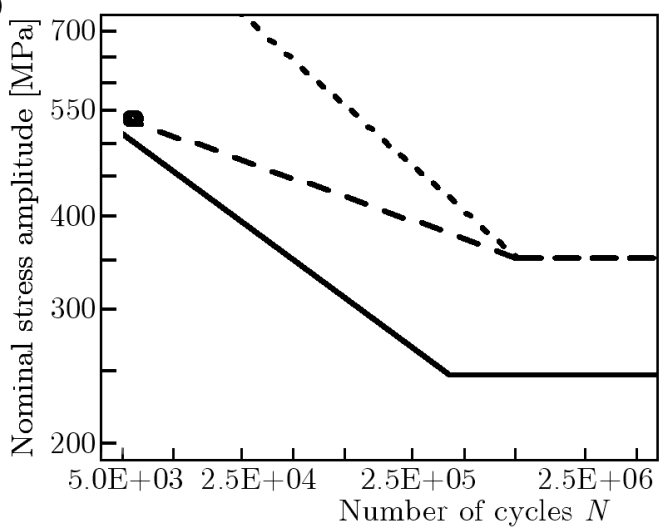

Fig. 10. Characteristics for: (a) material C45+C, smooth sample, (b) material 42CrMo4, smooth sample, (c) material C45+C, notched sample, (d) material 42CrMo4, notched sample; solid line experimental characteristics, dotted line - FITNET method, dashed line - FITNET method with experimental support

The proposed method determining the material life is emploed to make the computation using the foregoing equation based on the following relationship

$$
N=\left(\frac{Z}{\sigma_{a}}\right)^{\frac{\log \frac{N_{0}}{400\left(R_{e} R_{m}\right)^{-10}}}{\log \frac{0.9 R_{e}}{Z}}} N_{0}
$$

For the notched samples, the method of determining the life is expressed as follows (Strzelecki and Sempruch, 2013)

$$
N=\left(\frac{Z_{k}}{\sigma_{a}}\right)^{\frac{\log \frac{N_{0}}{10^{3}}}{\log Z+\frac{\log \left(0.9 R_{e} Z Z\right)}{\log \left(N_{0} / N_{R} R^{e}\right)} \log \frac{N_{0}}{10^{3}}-\log Z_{k}}} N_{0}
$$

The values of errors depending on the quantity of experimental information for the tested materials are shown in Fig. 11. Figure 11 illustrates the determination of the relative error using the following formula

$$
\xi_{p}=\left|\frac{N_{e}-B_{p}}{N_{e}}\right| \cdot 100 \%
$$

The time required for completing the measurement is assumed as the time of the experiment. The times for the preparation of measuring instruments, preparation of samples, etc. are not taken into account. It is assumed that the measurement of hardness would take 10 minutes (A) 


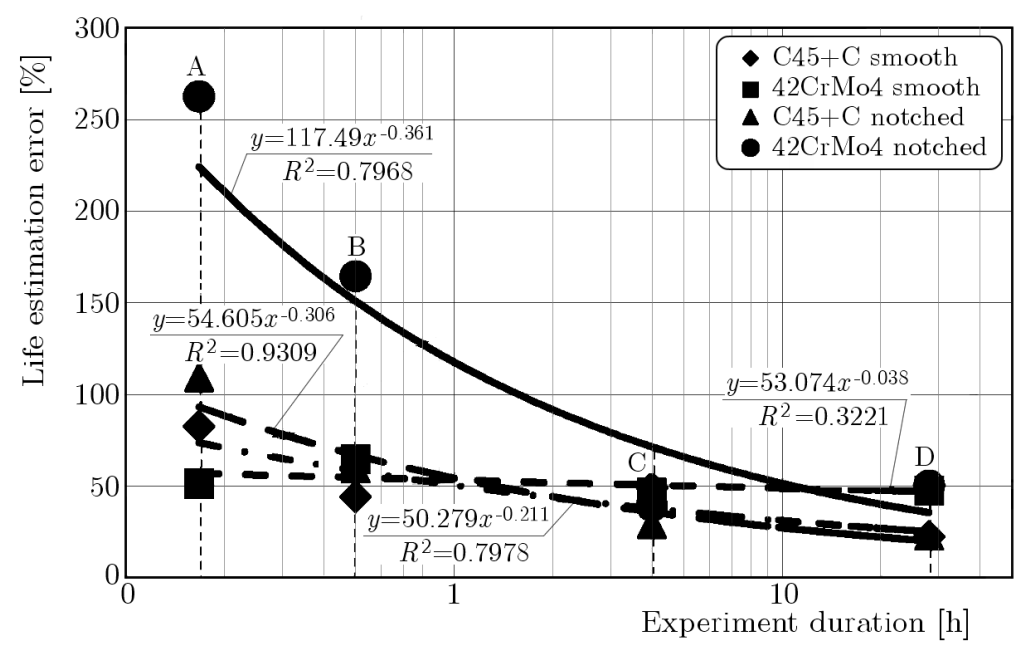

Fig. 11. Error of the analytical method error depending on the number of quantities determined experimentally

and the static tensile test 30 minutes (B). But the determination of life for a limited strength time for 3 samples takes 4 hours (C). For the experiment carried out using the Locati method, a 24-hour measurement time (D) is adopted.

\section{Summary and conclusions}

The verification of the methods for analytical determination of fatigue characteristics presented in Section 2 demonstrated that the value of the error can be 3 times larger than the experimental value. Further, the inaccuracy of determining the fatigue life using these methods can lay on either the safe or unsafe side with equal probability. Even if the proposed method provides better verification results, the accuracy of life determination carries a significant error. In addition, Section 5 presents the verification of the analytical method using the reliability coefficient $C_{R}$, which makes it possible to obtain characteristics featuring the desired probability. Based on the characteristics obtained, the results are satisfactory except for the plot for material $42 \mathrm{CrMo} 4$ and notched samples.

In order to improve the estimation of the fatigue life, the authors propose the analytical-and-experimental (hybrid) method. The experimental verification demonstrated that the error made while using this algorithm combined with laboratory testing can be significantly smaller, which is shown in Section 5. Therefore, whenever it is possible to conduct an experiment, it is recommended that the fatigue life is determined experimentally for 3 samples within the limited life range defined in Section 3.

\section{References}

1. Boyer E., 2003, Atlas of Fatigue Curves, Boyer E. (edit.), 5-th ed., American Society for Metals, Ohio

2. Jakubiec W., Malinowski J., 1996, Metrology of Geometrical Dimensions (in Polish), 2-nd ed., Warszawa WNT

3. Koçak M., Webster S., Janosch J.J., Ainsworth R.A., Koers R. (edit.), 2006, FITNET Fitness-for-Service PROCEDURE - FINAL DRAFT MK', Vol. I: FITNET FFS Procedure, European Fitness-for-Service Thematic Network - FITNET 
4. Lee Y.-L., Pan J., Hathaway R.B., Barkey M.E., 2005, Fatigue Testing and Analysis, University of Alabama, Elsevier

5. Ligaj B., Szala G., 2010, Experimental verification of two-parametic models of fatigue characteristics by using the tests of S55J0 steel as an example, Polish Maritime Research, 17, 1, 39-50

6. PARK J.H., Song J.H., 1995, Detailed evaluation of methods for estimation of fatigue properties, International Journal of Fatigue, 17, 5, 365-373

7. Robak G., Szymaniec M., Łagoda T., 2012, The fictitious radius as a tool for fatigue life estimation of notched elements, Material Science Forum, 726, 27-32,

8. Schijve J., 2009, Fatigue of Structures and Materials, 2-nd ed., Springer

9. Sempruch J., Strzelecki P., 2011, Error of fatigue life determinated according to the FITNET method, Engineering Mechanics, 531-534

10. Skibicki D., Sempruch J., Pejkowski L., 2012, Steel X2CrNiMo17-12-2 testing for uniaxial, proportional and non-proportional loads as delivered and in the annealed condition, Material Science Forum, 726, 171-180

11. Stephens R.I., Fatemi A., Stephens R.R., Fuchs H.O., 2001, Metal Fatigue in Engineering, 2-nd ed., New York Wiley

12. Strzelecki P., Sempruch J., 2012, Experimental verification of the analytical method for estimated S-N curve in limited fatigue life, Material Science Forum, 726, 11-16

13. Strzelecki P., Sempruch J., 2013, Experimental verification of the analytical method for determining the S-N curve for alloy steel, Key Engineering Materials, 598, 219-224

14. Tomaszewski T., Sempruch J., Piątrowski T., 2014, Verification of selected models of size effect based on high-cycle fatigue testing on mini specimens made of EN AW-6063 aluminium alloy, Journal of Theoretical and Applied Mechanics, 52, 2, 883-894 